\title{
TTR
}

Traduction, terminologie, re?daction

\section{Babel : avant, après}

\section{Alexis Nouss}

Volume 3, numéro 2, 2e semestre 1990

La traduction des textes sacrés : le domaine biblique

URI : https://id.erudit.org/iderudit/037068ar

DOI : https://doi.org/10.7202/037068ar

Aller au sommaire du numéro

\section{Éditeur(s)}

Association canadienne de traductologie

\section{ISSN}

0835-8443 (imprimé)

1708-2188 (numérique)

Découvrir la revue

\section{Citer cet article}

Nouss, A. (1990). Babel : avant, après. TTR, 3(2), 53-70.

https://doi.org/10.7202/037068ar d'utilisation que vous pouvez consulter en ligne.

https://apropos.erudit.org/fr/usagers/politique-dutilisation/ 


\title{
Babel: avant, après
}

\author{
Alexis Nouss
}

«Ayant été la tranchante et définitive réponse, le sacré est muet. Il se situe avant et après la question.»

Edmond Jabes

Dans sa célèbre étude sur le cas du Président Schreber, Freud rapporte comme élément des plus intéressants la Grundspräche, langue fondamentale, originelle, première, qui serait celle de Dieu et dont celui-ci se servirait pour communiquer avec les âmes purifiées. Le Président Schreber l'a apprise et si on en croit ce qu'en dit Freud, il s'agit d'un allemand assez classique et parfois grossier. Or, curieusement, Freud ne s'y attarde pas dans son analyse alors que, dans le post-scriptum de l'ouvrage, il procède pourtant à l'une de ses premières incursions dans le domaine de la mythologie, champ d'études naturel d'un tel phénomène. Il y reviendra brièvement dans la dixième conférence de son Introduction d la psychanalyse à propos du symbolisme des rêves (1975).

L'analogie serait cependant facile à établir entre cette langue fondamentale et la couche psychique première que Freud entend mettre au jour. Par ailleurs, un simple intérêt biographique aurait pu pousser le futur auteur de Moüse et le monotheísme à se pencher plus attentivement sur ce langage mythique.

Sans épiloguer davantage sur le silence de Freud ici, nous voudrions retenir la Grundspräche pour la pertinence de sa description dans une perspective traductologique. Il est en effet aisé de repérer un double aspect fondamental dans la langue du Président Schreber que nous pourrions définir comme un mouvement général 
de rétrécissement inter-linguistique: passage d'une langue compréhensible à une langue ésotérique, d'une part, et passage d'une langue commune à une langue réservée à une élite, d'autre part. Or il est tout aussi aisé de renverser ce mouvement pour définir ce qui serait le mouvement même du traduire, à savoir le passage d'une langue (ou d'un texte) ésotérique, réservé à quelques-uns, à un message clair et ouvert à tous. En termes psychanalytiques, nous pourrions parler du passage d'une langue maternelle, exclusive et individuelle, à un langage (je passe à dessein de langue à langage) ordonné, symbolique et communicateur, un langage paternel, tout à fait adéquat à l'énoncé d'un «Nom-du-Père», dirait un lacanien.

Cette notion de langue fondamentale n'est pas inconnue dans l'héritage culturel occidental. George Steiner rappelle qu'une réflexion de type gnostique, principalement portée par la tradition occulte, sur l'existence d'une «langue originelle unique ou $U r$ Sprache» (1978, p. 66) nourrit toute une philosophie linguistique jusqu'au XVIII' siècle, se perpétuant au-delà chez Walter Benjamin, Kafka et Borges. Cette langue, pour des raisons liées à l'histoire et à la sociologie des religions révélées, a souvent été associée à l'hébreu:

De la Genèse II, 2 aux Investigations philosophiques de Wittgenstein et au premier article inédit de Chomsky sur les morpho-phonèmes de l'hébreu, la pensée juive a joué un rôle majeur dans la mystique, l'érudition et la philosophie linguistiques. Pour les Juifs comme pour les gentils, les livres mosaïques possèdent un caractère de révélation étranger à tout autre corpus postérieur. L'hébreu a servi à maintes reprises à éprouver les autres langues. L'herméneutique juive fournit les têtes de chapitres qui orienteront la réflexion occidentale sur l'essence des langues humaines et l'énigme de leur mise en pièces (Ibid., pp. 67-68).

"Adamique», a-t-on également pu dire de cette langue première dans le lexique ésotérique. A double titre, car ce serait celle qui à la fois aurait façonné le premier homme puis qui serait entrée en sa possession afin qu'il en désigne les choses du monde. «Le parler de l'Éden était du verre le plus pur; étincelante, la compréhension absolue le traversait à flots», écrit Steiner (Ibid., p. 66). Cette croyance table en effet sur une parfaite adéquation entre les mots et les objets. Maître de cette langue et l'épousant, l'humain rayonnerait dans un équilibre ontologique sans failles et son rapport au monde ne rencontrerait une réalité aucunement probléma- 
tisée. Existence sans histoires (puisque avant l'histoire), conscience sans ombres, peut-on dès lors véritablement parler de langage dans ce royaume de la compréhension immédiate et absolue? On comprend en tous cas qu'inévitablement se pose la question du sens, de sa production et de sa réception en dehors d'un véhicule prenant en compte le devenir même de l'expérience humaine.

Ce qui semble en jeu ici est la croyance en l'existence, audelà ou plus exactement en-deçà de sa manifestation linguistique, d'un noyau de sens qui serait fondamental, énoncé primal qui contaminerait de sa primauté son énonciation. Incommunicable, intransmissible sinon par l'initiation ou l'effusion mystique, ce foyer irradiant dessine aussitôt le soupçon de l'intraduisibilité. «Le noyau du texte original est intouchable par la traduction, il y a de l'intouchable, c'est ça le sacré: le sacré dit: ne me touche pas» (1982), avance Derrida qui établit de même le lien avec ce qu'il nomme «une langue archi-maternelle» (Ibid). Sacré-intraduisible-langue première, triade conceptuelle qui nous situe au cœur du problème.

Walter Benjamin élaborant dans sa réflexion sur la traduction (1971) un concept proche de celui de langue première, die reine Sprache, la langue pure, on a tôt fait de confondre les deux notions et de les identifier à l'hébreu. C'est le cas notamment de Jean-René Ladmiral qui a consacré plusieurs articles à une critique de Benjamin et à la mise en place d'une articulation théologique du problème de la traduction. ${ }^{1}$ Il a pu ainsi écrire:

Historiquement, cet essentialisme platonisant des langues avait débouché sur des spéculations «étymo-logiques» (sic) cherchant à faire de l'hébreu, langue des Saintes Écritures, la langue-mère de toutes les langues. Cet horizon mysticothéologique est bien le point de fuite des réflexions de Benjamin, dont le dernier mot consiste à renvoyer la traduction au paradigme idéal de la Bible. (1988, p. 41)

Or cette approche nous semble être fondée sur un double postulat inscrit dans une lecture dont nous ne partageons pas les directions. Assimiler, d'une part, la «langue pure» de Benjamin à l'hébreu soulève certaines difficultés si l'on se reporte à l'essai de Benjamin sur la question et si l'on considère l'itinéraire biographico-intellectuel de Benjamin, tel qu'en témoigne notamment

1. Voir références. 
Gershom Scholem (1978). D'autre part, faire de l'hébreu cette «langue adamique» visée ou rêvée par les spéculateurs gnostiques n'est pas si aisé eu égard aux données mêmes de la tradition judaïque.

Faute de place, nous ne pourrons ici que discuter ce second point et tenterons donc de montrer que cette croyance d'une langue première dont la sacralité entraînerait l'intraduisibilité et le littéralisme n'est pas représentée dans la pensée hébrailque telle qu'elle s'exprime dans ses diverses sources: bibliques, talmudiques, midrachiques, kabbalistiques et philosophiques. La langue originelle y est au contraire perçue comme le foyer d'un appel constant à la traduction, braise qui ne peut irradier que d'être constamment caressée par de multiples souffles, le sens n'en étant jamais épuisé et ne pouvant se déployer précisément que dans cette sortie vers l'autre, que dans le mouvement du traduire. L'hébreu n'est pas la langue du secret, elle n'est que la langue du sacré (précisément ce que dit l'hébreu pour s'auto-désigner, et non pas «langue sacrée»)', le sacré compris comme l'invitation à sortir des langages particuliers pour se situer dans le vertige du dialogue, dans l'éclat (comme lumière et comme fragment) de l'entre-deux, le sacré comme espace à part (étymologie du mot hébreu désignant le sacré) qui est celui se déployant entre les êtres humains et où ceux-ci peuvent se rencontrer, espace où le monde devient humain parce qu'il est objet de dialogue, selon les termes de Hannah Arendt (1974, p. 34).

Dans cette perspective, nous donnons raison à Jean-René Ladmiral dans son affirmation d'un impensé théologique derrière les discours sur la traduction, en précisant toutefois que la théologie du judaïsme n'a rien de commun avec la métaphysique irrationnelle et «prélinguistique» dont ce théoricien nous entretient.

\section{Babel: avant et après}

Commençons par le commencement qui, dans notre présente perspective, ne peut être autre que genèse, le Livre de la Genèse. Et dans ce livre, allons directement au passage qui pose le problème de la traduction, évidemment l'épisode de la Tour de Babel pour trouver dans le passage sur la traduction une théorie de la traduction. La traduction littérale du verset décrivant la situation pré-

2. Nous devons cette remarque à Francine Kaufmann. 
babelienne (Gen. 11,1) donnerait: «Et toute la terre n'était qu'une langue unique et des paroles uniques.»

Langue unique que la traduction rabbinique identifie à l'hébreu, comme l'atteste le commentaire de Rachi, éminent maître du $\mathrm{XI}^{\circ}$ siècle qui vivait en France et dont les gloses font intimement partie de la lecture juive traditionnelle du texte biblique. Cet hébreu fondateur, cette langue fondamentale, l'est d'autant plus que le commentaire traditionnel en fait la langue employée à la Création et pour la Création (Rachi, sur Gen. 2,23).

L'identification devient en revanche problématique dans l'après-Babel où les cartes linguistiques semblent avoir été redistribuées dans un sens nouveau et où il devient difficile de faire de l'hébreu fondateur la langue particulière que nous connaissons aujourd'hui comme l'hébreu. Le changement peut aisément se comprendre comme une réorientation dans l'histoire, le passage à un autre mode d'être historique et corrélativement à un autre usage du langage que nous préciserons plus bas.

En effet, lors d'un autre épisode qui est comparé à celui de la Création, la Révélation sur le Mont Sinaï (le parallélisme est établi du fait que dix paroles ont crée le monde et dix paroles les fameux dix «commandements» d'une version commune mais erronée - ont été données au Mont Sinaii), il est dit dans plusieurs sources traditionnelles de la langue employée au cours de cette révélation qu'elle n'est plus une mais éclatée en soixante-dix langues, ce nombre étant symboliquement celui des langues du mon$\mathrm{de}^{3}$.

Et il ne s'agit pas d'une traduction polyglotte qui réintroduirait l'idée d'une langue fondamentale antérieure mais bien, dans la réception (qui est le seul niveau auquel s'intéresse véritablement la pensée juive: le texte toraïque parle le langage des hommes, selon l'adage talmudique), le dire qui se donne comme traduire. Preuve en est qu'une autre interprétation du verset de Jérémie fondant le précédent commentaire $e^{4}$ le comprend comme la possibilité de lire soixante-dix sens dans le texte biblique, ce qui établit qu'il n'y a pas qu'un sens premier et supérieur, de même qu'il n'y

3. Voir notamment Talmud de Babylone, Traité Chabbat, 88b.

4. Talmud de Babylone, Traité Sanhedrin, 34a. 
aurait pas une langue première et supérieure. Ce sont les soixantedix langues qui forment cette langue fondamentale.

Un développement similaire de cette idée se retrouve dans le commentaire d'un mot figurant en deux endroits du Deutéronome: «[...] Moïse commença à expliquer cet enseignement» (Deut. 1,5; notre traduction) et «Et vous inscrivez de manière très claire sur les pierres tous les mots de cet enseignements (Deut. 27,8; notre traduction). Le mot signifiant sous deux formes grammaticales différentes «expliquen» et «de manière très claire» est interprété comme voulant dire: «en soixante-dix langues». L'interprétation, rapportée par Rachi, figure dans le traité talmudique Sotah (32a) ainsi que dans les recueils midrachiques Tanhoumah et Rabah (Gen. 49).

Il s'agit encore ici de désigner un message qui, par la teneur de son énoncé, entraîne une énonciation dont l'homogénéité du vecteur linguistique est niée au profit d'une multiplicité expressive. Ce commentaire nous est très précieux, car il éclaire et illustre le concept de reine Sprache de Benjamin:

Toute parenté supra-historique entre les langues repose bien plutôt sur le fait qu'en chacune d'elles, prise comme un tout, une chose est visée, qui est la même, et qui pourtant ne peut être atteinte par aucune d'entre elles isolément, mais seulement par le tout de leurs visées intentionnelles complémentaires; cette chose est le langage pur, die reine Sprache. $(1971,266)$

Ce que Giovanni Gentile, dans un texte sur la traduction antérieur de quelques années à celui de Benjamin, a pu aussi exprimer en disant: «Nous ne traduisons jamais car, quelles que soient les langues, chacune prise comme telle n'en forme pas plus d'une, et, toutes ensemble, elles n'en forment qu'une seule.» (1988, p. 17).

Il est clair maintenant que la référence à une langue première, la «langue pure» de Benjamin ou l'hébreu d'après Babel dans le commentaire juif, ne doit pas être comprise comme l'affirmation d'un noyau linguistique insécable, homogène et transcendant, mais l'indication d'une structure dynamique se dévoilant dans l'histoire. Que s'est-il alors passé à Babel? Qu'est-ce qui a entraîné l'hébreu à devenir langue du multiple et du dialogue, langue «multilogue» en quelque sorte? 
La narration biblique évoque la construction de la tour atteignant le ciel, symbole de l'union et de l'orgueil des humains, et le courroux d'une divinité que l'on pourrait croire simplement jalouse de ses prérogatives, à l'image des tragi-comédies du Panthéon grec. Mais, à rester dans une lecture qui voit dans la Bible plus le récit d'une écriture que l'écriture d'un récit, on pourrait avancer que ce que l'hébreu rencontre à Babel, ce qui le fait passer de «langue une» à «langue plurielle», c'est le silence. Non le contraire de la parole mais un certain état de la parole, une chrysalide du dire, un verbe non énoncé.

Un silence qui toucherait à l'absolu du dire pour George Steiner et qui déborderait donc la vocation humaine:

Il n'est pas impossible qu'on ait interpreté à tort le mythe de Babel. La tour ne coïnciderait pas avec la disparition d'un monisme privilégié, d'un étaty d'universalité linguistique. L'affolante prodigalité des langues existait depuis longtemps et compliquait l'exécution des entreprises humaines; c'est en essayant d'édifier la tour que les nations sont tombées sur le grand secret: la compréhension véritable n'existe qu'avec le silence. Elles se sont mises à bâtir sans un mot, et c'est là qu'était le danger qui menaçait Dieu. (1978, pp. 267-268)

Un silence qui précéderait l'histoire pour Henri Meschonnic et serait donc en mal d'enfanter l'aventure humaine:

Paradoxalement, le mythe théologique qui associe, et solidarise, la dispersion des peuples et la pluralité des langues, fonde 1'histoire empirique. Et la «langue une» d'avant Babel peut apparaître non plus comme la «langue de sainteté» mais comme l'absence du langage précédant le langage. [...] Parce que l'historicité du langage, qui n'est pas séparable de celle des langues, est liée à leur diversité. $(1985$, p. 27)

\section{Traduire Babel}

Cette dynamique polyglossique explique rétroactivement l'épisode de Babel dans une optique nouvelle qui le valorise positivement. «Traduire Babel», écrivons-nous en intertitre, aux deux sens: traduire le passage biblique de la Tour de Babel et comprendre dans 
une perspective traductologique l'enseignement que peut nous apporter cet épisode, et ce en nous appuyant sur l'œuvre traductologique méconnue d'André Nehers.

L'humanité pratiquait donc une langue unique, selon notre traduction littérale, mais le verset apporte aussi cet ajout intrigant: «et des paroles uniques». Ce dernier terme expose le drame qui va entraîner l'épisode de Babel. «Paroles uniques» reçoit diverses interprétations dont le commentaire de Rachi en rapporte trois: des paroles qui traitent de l'unicité divine, des paroles «dures» (selon un rapprochement de racines) et la suivante, d'une étonnante modernité: paroles uniques parce qu'elles participaient d'un plan, d'une stratégie unique. Autant dire qu'elles sacrifiaient à une idéologie qui serait aujourd'hui qualifiée de totalitaire. C'est sur cette base qu'André Neher propose la traduction suivante: «Le malheur, c'est que l'humanité, dans sa totalité, était d'un seul bord et vivait une seule histoire.» $(1969$, p. 49) Nous sommes loin du «Toute la terre avait une même langue et des paroles semblables» de la version officielle juive de la Bible du Rabbinat. Le texte de Neher nous dessine immédiatement l'horizon sémantique du verset hébraïque, à savoir une histoire sans historicité, où l'expérience individuelle ne serait pas prise en compte.

André Neher justifie sa traduction par un exposé de sa méthode dont il serait important de présenter un aperçu avant de revenir à sa proposition. Après avoir rappelé combien les traductions bibliques étaient événements fondateurs pour les cultures et les langues (la Septante, la Bible de Luther, la Version autorisée en langue anglaise, etc.), Neher situe sa démarche à la lumière des essais de Buber et Rosenzweig, Harris Orlinsky et Edmond Fleg. II dégage trois règles principales, trois «lois» (Ibid., p. 30 sq.): la «loi de l'haleine» (expression empruntée à Rosenzweig) qui brise le découpage médiéval en versets et retrouve les rythmes propres au texte en fonction de sa logique et des commentaires traditionnels; la «loi de la fidélité horizontale» basée sur le respect des termes

5. Henri Meschonnic condamne la traduction d'André Neher comme "glose substituée au texte» et «effet désastreux produit par l'équivalence dynamique» (1985, p. 19). Nous souscrivons à sa critique dans la perspective qui est la sienne, l'affirmation de la signifiance du rythme. Nous tenons cependant à intégrer ici le travail d'André Neher pour sa dimension herméneutique et l'interprétation que sa traduction donne du mythe de Babel. 
identiques qui permet d'exprimer l'unitế et la cohérence du texte biblique, d'en «relier les fils» et enfin la «loi de la fidélité verticale», qui consiste à choisir entre toutes les strates sémantiques des termes bibliques celle qui offrira la signification la plus pertinente en secouant la rigidité lexicale des conventions. Il écrit:

Si Aristote, Saint-Paul, Catherine de Bore, Lessing ont inspiré à la Septante, à la Vulgate, à Luther, à Mendelssohn la détection d'un niveau précis du sens biblique, il est légitime que Kierkegaard, Karl Marx, Freud et Kafka inspirent des détections nouvelles et différentes aux traducteurs du $\mathrm{XIX}^{e}$ et du $\mathrm{XX}^{e}$ siècle. La valeur intrinsèque de la traduction est à ce prix, à condition toutefois que l'actualisation du langage soit précédée d'une exploration verticale du langage biblique, qui n'hésite pas à aller jusqu'au fond de ses virtualités historiques et traditionnelles. (Ibid., p. 36)

C'est en fonction de la double loi de fidélité que Neher substitue «l'humanité dans sa totalité» à «toute la terre», «un seul bord» à «langue unique» et «vivait une seule histoire» à «paroles uniques». On reconnaît aisément des accents philosophiques très contemporains et, les retrouvant dans la suite du passage tel que Neher l'a traduit, c'est une toute nouvelle compréhension de Babel qui surgit:

6. Le premier mot de Dieu fut l'approbation: «Oui, dit-Il, ils forment un seul peuple, parlant une seule langue, et leur idkal, c'est ce qui peut leur être utile au niveau collectif. C'est bien cela l'ambivalent pouvoir créateur de l'homme. Sur ce plan de l'histoire, rien ne leur sera plus impossible de ce qu'ils projetteront depuis les sources de leur créativité;

7. mais, sur l'autre plan, nous participerons objectivement à leur histoire et là, nous ramènerons leurs langages à leur forme la plus naîve, afin qu'ils restent prochains et solidaires, même en ne se comprenant plus.»

8. C'est donc à partir de là que Dieu les fit se refléter dans les visages de l'humanité entière. Comme première conséquence, ils cessèrent de construire la ville.

9. C'est la raison pour laquelle on lui donna le nom de Babel, car c'est littéralement là que Dieu pétrit la pâte de la langue de l'humanité entière, et c'est de là qu'Il les fit se refléter dans les visages de l'humanité entière. (Ibid. pp. 49-50) 
Ce que nous apprend cette traduction, c'est que le texte biblique ne légitime d'aucune manière le découpage classique de certains théoriciens entre un avant et un après Babel, avec les valorisations qu'il implique. Nulle malédiction, nul péché originel mais un devenir nouveau offert à l'humanité. Babel n'introduit en fait qu'une nouvelle modalité, un nouvel usage de la langue, une «intentio» au sens de Walter Benjamin au sein d'une langue qui était figée dans une unicité stérile. Babel introduit le dialogue dans l'usage de la langue - et donc le visage de l'autre - introduit le traduire à l'intérieur du dire. La traduction du dernier verset, 9, du passage le souligne: il y est question d'une seule langue qui n'est que «babélisée», à savoir touchée, pétrie, modifiée, rendue hététogène. Par ailleurs, rappelons-nous que la dispersion n'est pas un châtiment puisqu'elle était prévue dès le récit de la Création: «Multipliez-vous et remplissez la terre.» (Gen. IX, 1)

Introduire dans le dire. La littérature talmudique offre de nombreux témoignages de cette orientation fondamentale de la pensée philologique juive. J'ai montré ailleurs (1989) comment la révélation était dans la tradition talmudique inséparable de sa/la traduction. Je voudrais ici citer d'autres exemples prouvant combien, à l'autre pôle d'énonciation, la réception de cette révélation, ce qu'il est légitime d'appeler sa lecture, est tout aussi inséparable de sa/la traduction.

\section{Sainte traduction}

Dans le traité talmudique Chabbat portant sur les problèmes et les lois relatifs au jour du repos, aux folios 115 et 116 , nous trouvons une discussion fascinante sur la question suivante: On sauve les livres saints en cas d'incendie. Mais que fait-on s'ils sont écrits en d'autres langues que l'hébreu? Leur statut de traductions les situe-til au même rang? La réponse globale que je livre ici sans en préciser les prolégomènes est positive. De même, si elles deviennent impropres à l'usage, les traductions doivent, comme les livres saints, être enterrées, et non détruites, procédé réservé à des objets de statut inférieur. Et cette réponse positive a d'autant plus de poids qu'il est aussi précisé (115b) que des amulettes, même si elles contiennent des noms divins et des passages du Pentateuque, ne doivent pas être sauvées et «doivent brûler là où elles se trouvent». Le traduit, pour reprendre un terme proposé par Jean-René Ladmiral, puisqu'il appartient au livre, c'est-à-dire à ce qui véhicule le sens, a plus de valeur que l'hébreu de l'amulette qui n'est plus 
qu'une langue morte, au sens propre, puisque le vivant, l'être humain lecteur, n'a plus part à son énonciation.

On retrouve cette affirmation de l'importance primordiale du traduit dans deux passages étonnants du traité Berakhot traitant des Bénédictions. Au folio 8, la discussion porte sur les textes qui sont intégrés dans la lecture publique. Il y est dit que celle-ci comporte deux fois la section hebdomadaire du Pentateuque en hébreu et une fois la traduction en araméen (celle-ci étant le paradigme du traduit, comme je l'ai montré, op. cit.), y compris «les passages Ataroth et Dibon", ces derniers étant des listes de noms qui ne sont pas traduits dans le Targoum, la traduction en araméen. Si le Talmud se donne la peine d'apporter cette précision qui peut sembler inutile, c'est bien pour insister sur la valeur intrinsèque de la traduction. égale importance:

$\mathrm{Au}$ folio $45 \mathrm{a}$, nous trouvons le passage suivant, d'une

R. Simeon b. Pazzi a dit: D'où apprenons-nous que celui qui traduit [celui qui traduit la section lue en lecture publique] ne doit pas avoir un niveau de voix plus élevé que celui qui a lu? Parce qu'il est écrit: Moïse parlait et Dieu lui répondait par une voix (Ex. XIX, 19). Les mots «par une voix» étaient inutiles. Ils signifient: par la voix de Moïse. On a de même enseigné: le traducteur ne doit pas élever sa voix au-dessus de celle du lecteur. [Notre traduction]

Passage déconcertant puisque, dans le contexte, nous sommes amenés à considérer Dieu comme un traducteur. Les commentateurs connus sous le nom de tossaphistes expliquent qu'il s'agit d'une image: Dieu abaissait la puissance de sa voix pour qu'elle soit audible. Mais on peut y lire deux autres significations: Dieu, dans sa révélation à Moïse, traduisait effectivement une Torah première mais divine pour qu'elle puisse être reçue dans la dimension et la conscience humaines. On peut aussi comprendre dans une perspective traductologique: la voix du traducteur ne doit pas être plus élevée que celle du lecteur parce qu'une traduction n'épuise jamais définitivement le sens du texte, ne le recouvre pas, en somme.

Il est pertinent de mettre en correspondance cette image et l'interprétation que je viens de lui donner avec un passage de 
l'essai de Walter Benjamin sur la traduction en gardant en mémoire l'idée de la voix du traducteur ne recouvrant pas celle du lecteur: «Une traduction véritable est transparente; elle ne recouvre pas l'original, n'en retient pas l'éclat mais permet à la langue pure, comme raffermie dans sa propre énonciation, d'irradier encore plus brillamment à travers l'original.»[Notre traduction]

De l'auditif au visuel, l'idée est la même que dans le Talmud. Or la transparence est un concept que Jean-René Ladmiral utilise par opposition à celui de dissimilation. Mais il n'en est pas partisan et écrit ainsi à propos de Benjamin:

A faire entrer, nécessairement de force, ce que Mallarmé appelle «les mots de la tribu» dans une syntaxe qui leur est étrangère, la traduction devient une sorte de marquetterie langagière qui fait violence à la langue-cible. [...] Et Benjamin sait gré à de grands traducteurs comme Luther et Hölderlin justement, mais aussi Voss ou George, d'avoir travaillé sur la langue, de l'avoir transformée, d'avoir «élargi les frontières de l'allemand». Il s'agit en effet d'œuvrer au mouvement de la langue (Sprachbewegung) et d'y retrouver en filigrane (ou «entre les lignes») les «fragments d'un langage plus grand», de ce «langage pur» qui fait d'autant plus penser au vieux rêve mythique d'une langue adamique que la référence aux «versions interlinéaires» réinvestit le langage oublié qu'est l'hébreu-source des textes sacrés d'une dignité que, seule, la litote d'une sécularisation matérialiste sinon athée exige de passer sous silence... (1981, p. 74)

Outre que si la langue-cible pouvait être violentée reviendrait à reconnaître à celle-ci - et à toute autre - une "pureté" que justement il ne veut reconnaître, Benjamin ne se réfère pas à cette langue adamique consacrée par les spéculations ésotériques et mystiques. Celle-ci appartiendrait au mode du secret que nous évoquions plus haut, un énoncé statique et préservé, précisément un hébreu-source qui se voudrait source parce qu'hébreu. Les exemples donnés à partir des textes talmudiques prouvent assez le contraire. L'hébreu ne se veut jamais source mais lieu de confluences, delta peut-être. Jean-René Ladmiral développe là une lecture de Benjamin qui voit en celui-ci un littéraliste sacrifiant à un «anticommunicationalisme linguistique» (Ibid., p. 72). Il est difficile - pour n'avancer qu'un seul argument - de concilier cette position avec la philosophie de l'histoire de Benjamin qui nie la linéarité sourde 
pour prôner une historicité qui permet le dialogue des langages et des expériences. Le littéralisme présumé de Benjamin «opte pour le mot contre les paroles du texte, pour les mots de la langue contre la Parole», selon Jean-René Ladmiral (Ibid., p. 71). Les mots que défend Benjamin ne sont pas ceux du mot-à-mot ni du calque. Au contraire, son essai sur le traducteur est un plaidoyer pour l'ouverture à ce qui dans les mots relève de l'intentio, à ce qui dépasse à la fois les mots et le sens exact (cf. Meschonnic, 1986), appel à l'émotion, à la connotation, à ce qui dans le langage en appelle à un autre langage, à une complémentarité, à un désir en un mot.

Un passage de l'essai de Benjamin qu'aime à citer JeanRené Ladmiral est le suivant: «car la phrase est muraille dressée devant l'original, et la littéralité est arcade»(Ibid.). Cette dernière image évoque irrésistiblement un texte de Kafka, Vor dem Gesetz, le fameux «Devant la Loi» dans lequel un homme se voit interdire l'entrée de la Loi par un gardien pour, à la fin de sa vie passée dans l'attente, se faire dire que cette entrée lui était réservée. Je me suis autorisé ailleurs ${ }^{6}, m^{\prime}$ 'appuyant sur la souplesse sémantique de l'allemand, à traduire ce titre par «Devant» ou plutôt «Avant le verset», c'est-à-dire «Avant l'écrit» et même «Avant le traduit» et à traiter le texte comme support à une théorie de la traduction. On ne parvient jamais à la loi comme on ne parvient jamais à une traduction définitive mais être devant, c'est déjà en participer. Et cela parce que le vor allemand ne marque pas une rupture comme le «devant» français mais bien un rapport dynamique. Et traduire exprime ce rapport. Comme l'écrit Henri Meschonnic à propos du concept de force qui apparait dans le texte de Cicéron sur l'interpres et l'orator:

[...] le discours comme système de subjectivité dans le langage. La force suppose un sujet. C'est-à-dire un transsujet: le passage même du langage entre sujets. Ainsi le discours est de l'ordre du continu. La langue, avec ses unités, est de l'ordre du discontinu. Les distorsions à l'usage, s'il y en a, sont les éléments de ce système et de ce continu du discours. Alors seulement il y a une poétique du traduire, comme il y a la poétique d'une écriture. Et une traduction peut être la métaphore d'un texte. (1986, p. 79)

6. «Vor dem Gesetz: devant la loi, avant la langue. (D'une théorie de la traduction chez Kafka)», à paraître. 
La métaphore ou la métamorphose, pour rester avec Kafka. Die reine Sprache, la langue ou le langage pur, dont parle Benjamin, n'est pas une langue pure d'immanence et de méditation, une langue vide, mais une langue qui s'ouvre sur le vide pour que $s^{\prime} y$ déploient tous les jeux de la signifiance, pour que s'y rencontrent toutes les langues ou plutôt toutes les énonciations, et tous les sujets en leur histoire. Nous pourrions ici citer longuement l'essai de Benjamin en ce sens. Mais, pour revenir à la version interlinéaire de l'Écriture qui, comme on le sait, conclut son essai comme «idéal de toute traduction», celle-ci est choisie parce qu'elle est grosse de toute l'épaisseur sémantique de l'original, «sans la méditation de la signification». Benjamin écrit: «De même que dans l'original, le langage et la révélation ne font qu'un, de même la traduction ne doit faire qu'un avec l'original sous la forme de la version interlinéaire.» (1971) Or la révélation est précisément cette dynamique qui appelle à une réception constante, c'est-à-dire une lecture/interprétation en constant renouvellement.

\section{Ouverture du sacré}

Cet espace de dialogue et d'ouverture au(x) sens, cette version interlinéaire, le Talmud la reconnaît et la pratique continuellement comme une des énonciations de son discours. Des dizaines de passages de traités talmudiques montrent comment la discussion rabbinique invoque la traduction en araméen pour expliciter le sens de versets bibliques. La construction de ces passages est la suivante: telle opinion s'appuie sur tel verset pour se légitimer mais ce verset est difficilement compréhensible. On se réfère alors à sa traduction pour en saisir le sens et donc autoriser l'opinion en question. On renverse donc le mouvement du traduire pour investir d'autorité le traduit et non le texte biblique. De même, nombre de commentateurs citent la traduction pour appuyer leurs commentaires.

La tradition kabbalistique ne saurait manquer d'être aussi mentionnée, ne serait-ce que parce que sa présence pèse sur la mystique du langage dans laquelle Benjamin semble s'inscrire sous la double influence du romantisme allemand et de son amitié avec Gershom Scholem. Nous ne pourrions dans le cadre de cet article traiter de la philologue kabbalistique et ne ferons que citer brièvement Gershom Scholem: 
Attendu que toutes les langues sont nées d'une corruption du langage originel sacré au sein duquel le monde des noms originels se décompose immédiatement, elles sont encore directement liées à lui. Puisque toute langue a son centre dans le nom divin, elle peut lui être rapportée. Comme le dit Aboulafia, le mystique restitue la langue sacrée en procédant à une refonte générale de toutes les langues, si bien que chaque discours articulé qu'il prononce se compose à ses yeux des vingt-deux lettres sacrées $(1983$, p. 96).

De ce passage par la traduction pour quêter ou provoquer le sens, le Talmud offre un autre exemple dans le Traité Houlin au folio $139 \mathrm{~b}$ où la discussion cherche à trouver un enracinement biblique au personnage de Mardochée, le héros du livre d'Esther, Mordekhaï en hébreu: «Où le trouve-t-on? Comme il est écrit: Mor deror, Mar dakhya: myrrhe de liberté, myrrhe pure.»

J'ai évoqué dans mon article déjà cité la discussion du traité Meguila ${ }^{7}$ sur la possibilité de traduire où le grec est privilégié, justement parce que langue de traduction en raison de son universalité, de sa visée à l'universellement compréhensible. Comme l'écrit Marc-Alain Ouaknin, la traduction «n'est pas une possibilité mais une nécessité. La traduction serait une chance pour l'écriture. Après avoir sombré dans l'écrit, dans les «langues mortes», dans le dit, la traduction serait le premier dédire» (1989, p. 189). Et Ouaknin de remarquer que la traduction en grec est contemporaine de la loi orale, de la canalisation de la loi orale qui assure la vitalité de la lecture et du sens. La dynamique est la même: il s'agit de ne pas faire auvre morte, ni lettre morte, ni sens figé, c'est-à-dire tout autant mort, comme l'explique Meschonnic (1986), mais toujours viser au débordement, à l'au-delà du sens, là où un sujet rencontre son histoire et l'histoire de l'autre, miracle et mystère du langage. Benjamin consacre un développement de son essai sur la traduction au lien intrinsèque et indéfaisable entre les concepts de vie et d'histoire. C'est l'historicité entendue comme inscription active et non passive dans l'histoire qui est garante de vie, non la nature de l'âme. Or cette histoire est faite de différences. Maurice Blanchot développe merveilleusement cette idée du traducteur comme «maître secret de la différence des langues»

7. Voir aussi l'article de Francine Kaufmann dans le présent numéro. 
(1971, p. 71). La traduction, dit-il, rend visible ce qui sera toujours autre dans une langue. A rapprocher brutalement du propos du maître hassadique Rav Nahman de Breslev rapporté par Ouaknin: «La perfection de l'hébreu réside dans son imperfection et dans sa nécessité à être traduit en araméen.» $(1989$, p. 191) Quand il est dit que la perfection de l'hébreu tient dans sa possibilité et sa nécessité à être traduit, on peut facilement remplacer perfection par sacré. L'hébreu, parce qu'appelant la traduction, serait langue du sacré, jamais du secret.

En ceci, il est traductible. Il est même paradigmatique de l'acte de traduction. Benjamin pose que la dimension qui rend un texte traduisible, qui fait qu'il appelle la traduction, c'est ce qu'on peut appeler avec Derrida «sa structure survivante» (1982), ce qui en lui vise à la survie, non la conservation figée, le mausolée, l'embaumement, mais ce qui en lui maintient la vie, au-delà du physique, au-delà de la matérialité du texte, au-delà de l'histoire même de ce texte mais non au-delà de son historicité, lieu où des sujets pourront s'investir et dialoguer: «überleben», dit Benjamin. Peut-on oublier ici que dans le récit biblique précisément, la vie, le don de vie fait par le divin lié à l'humain, est lié à la parole? De même dans un texte, ce qui est lié à la langue va le lier à la vie, à la survie, au traduire.

Ainsi pour en revenir à la langue pure, die reine Sprache, peut-être la Grundspräche du Président Schreber, elle ne revêt d'autre dimension sacrée que d'être langue. Comme le dit Derrida, ce n'est pas «une langue purifiée de quoi que ce soit, c'est ce fait d'une langue, une langue» (1982). Il ajoute:

Voilà ce que nous apprend une traduction, non pas le sens contenu dans un texte traduit, non pas ceci ou cela, mais qu'il y a de la langue, que la langue est de la langue et qu'il y a une pluralité de langues qui ont entre elles cette parenté d'être des langues. (Ibid., p. 164)

Propos bien benjaminien et raison pour laquelle il n'y a pas de noyau intraduisible sinon au niveau fantasmatique. Il n'y a pas de noyau parce qu'il y a de la langue, il n'y a que de la langue. La conceptualisation de la philologie juive ne va pas dans un autre sens. Souvenons-nous du verset biblique: "Toute l'humanité n'était qu'une langue unique». Toute l'humanité, c'est-à-dire la Création, née d'un fait de langage et tirant de là son sacré. D'où la nécessité du traduire. D'où Babel. 
Or la Création n'est selon la tradition mystique qu'affaire de noms divins. Le Talmud, repris plus tard par le folklore magique, traite des possibilités de création à partir de combinaisons de noms divins (tel le mythe du Golem). Ce sacré-là, en hébreu (mais quel hébreu?), peut donc engendrer du vivant, de l'histoire. Derrida avance que le sacré est sacré parce que nom propre, «il ne se laisse pas totalement devenir commun» $(1982,195)$. Posons le contraire: nom propre, le sacré tend à devenir commun, tend, au sens le plus général, à être traduit.

Le Président Schreber avait tort. Sa Grundspräche n'était pas la langue de Dieu. Mais le Président Schreber avait aussi raison: il fallait le traduire.

Université Concordia

\section{Références}

ARENDT, Hannah (1974). Vies politiques. Paris, Gallimard, coll. «Tel».

BENJAMIN, Walter (1971), «La Tâche du traducteur». Mythe et violence. Trad. M. de Gandillac. Paris, Denoël.

BLANCHOT, Maurice (1971). «Traduire». L'Amitié. Paris, Gallimard.

DERRIDA, Jacques (1982). L'Oreille de l'autre (Textes et débats avec Jacques Derrida, sous la direction de Claude Lévesque et Christie V. McDonald). Montréal, VLB éditeur.

FREUD, Sigmund (1975). Introduction d la psychanalyse. Paris, Payot, coll. «Petite Bibliothèque Payot».

GENTILÉ, Giovanni (1988). «Du tort et du droit des traductions». Cahier du college international de philosophie, $\mathrm{n}^{\circ} 6, \mathrm{pp}$. 13-20. 
LADMIRAL, Jean-René (1981). «Entre les lignes, entre les langues». Revue d'esthétique, nouvelle série, $\mathrm{n}^{\circ} 1, \mathrm{pp}$. 67-77.

(1986). «Sourciers et ciblistes». Revue d'esthétique, nouvelle série, $\mathrm{n}^{\circ} 12$, pp. 33-42.

(1988). «Les Enjeux métaphysiques de la traduction. A propos de Walter Benjamin». Cahier $d u$ college international de philosophie, $\mathrm{n}^{\circ} 6, \mathrm{pp} .39-44$.

MESCHONNIC, Henri (1986). «Alors la traduction chantera». Revue d'esthétique, nouvelle série, $\mathrm{n}^{\circ} 12, \mathrm{pp} .75-80$.

(1985). «Moïse: Au commencement». Les Tours de Babel. Mauvezin, Trans-Europ-Repress.

NEHER, André (1969). De l'hébreu au français. Paris, Klincksieck.

NOUSS, Alexis (1989). «L'Interdit et l'inter-dit: la traduisibilité et le sacré». TTR, Vol. II, $\mathrm{n}^{\circ} 1, \mathrm{pp} .75-88$.

OUAKNIN, Marc-Alain (1989). Lire aux éclats. Paris, Lieu commun.

SCHOLEM, Gershom (1983). Le Nom et les symboles de Dieu. Paris, Éditions du Cerf.

Paris, Calmann-Lévy.

(1978). «Walter Benjamin». Fidélité et utopie.

STEINER, George (1978). Apres Babel. Paris, Albin Michel. 